\title{
Coalbed Methane Production System Simulation and Deliverability Forecasting: Coupled Surface Network/Wellbore/Reservoir Calculation
}

\author{
Jun Zhou, ${ }^{1}$ Guangchuan Liang, ${ }^{1}$ Tao Deng, ${ }^{2}$ Shiwei Zhou, ${ }^{3}$ and Jing Gong ${ }^{4}$ \\ ${ }^{1}$ Southwest Petroleum University, Chengdu 610500, China \\ ${ }^{2}$ China National Petroleum Corporation Guangzhou Petroleum Training Center, Guangzhou 510510, China \\ ${ }^{3}$ Branch of China Petrochemical Marketing Co. Ltd., Jiangxi Yichun Oil Company, Yichun 336000, China \\ ${ }^{4}$ China University of Petroleum, Beijing 102249, China
}

Correspondence should be addressed to Jun Zhou; 201599010096@swpu.edu.cn and Guangchuan Liang; lgcdjr@163.com

Received 21 October 2016; Accepted 21 December 2016; Published 31 January 2017

Academic Editor: Bhaskar Kulkarni

Copyright (C) 2017 Jun Zhou et al. This is an open access article distributed under the Creative Commons Attribution License, which permits unrestricted use, distribution, and reproduction in any medium, provided the original work is properly cited.

\begin{abstract}
As an unconventional energy, coalbed methane (CBM) mainly exists in coal bed with adsorption, whose productivity is different from conventional gas reservoir. This paper explains the wellbore pressure drop, surface pipeline network simulation, and reservoir calculation model of CBM. A coupled surface/wellbore/reservoir calculation architecture was presented, to coordinate the gas production in each calculation period until the balance of surface/wellbore/reservoir. This coupled calculation method was applied to a CBM field for predicting production. The daily gas production increased year by year at the first time and then decreased gradually after several years, while the daily water production was reduced all the time with the successive decline of the formation pressure. The production of gas and water in each well is almost the same when the structure is a star. When system structure is a dendritic surface system, the daily gas production ranked highest at the well which is the nearest to the surface system collection point and lowest at the well which is the farthest to the surface system collection point. This coupled calculation method could be used to predict the water production, gas production, and formation pressure of a CBM field during a period of time.
\end{abstract}

\section{Introduction}

$\mathrm{CBM}$ is one of the most important sustainable energy for the strategy of sustainable development in the 21st century. China is abundant with CBM resource. About 36.81 trillion cubic meters is stored in depth of less than $2000 \mathrm{~m}$ under the ground in the field [1]. The wells are intensively distributed in the on-site CBM blocks. The gas production and pipeline operation parameters for undergoing construction project could be predicted by the integration of surface/wellbore/surface pipeline network to get closer to the actual production data, which optimizes and guides the CBM surface construction and improves the production to maximize the industry economic benefit. Over the past few decades, many scholars have been studying the integration of the oil and gas production system and several models have been put forward. Dempsey et al. [2] first studied the coupling of gas reservoir flow simulation and surface system simulation, which built the foundation of other relative research on the production system integration. Startzman et al. [3], Trick et al. [4], Litvak and Darlow [5], Coats et al. [6], Al-Mutairi et al. [7], and Guyaguler et al. [8] also put forward their models of the reservoir/wellbore/surface system integration afterwards. Startzman et al. [3] proposed a model of reservoirto-surface system coupled simulation, but this model only applied to the development of large offshore oil fields and the scope of application was narrow. Trick et al. [4] combined the black oil reservoir simulation software IMEX and the ground system simulation software FORGAS for forecasting the production of gas field. The coupling process of these two models is applicable to the coupling of any reservoir simulator with the ground system model which includes bottom-hole inflow dynamic curve and bottom-hole pressure loss calculation module. Litvak and Darlow [5] studied the 
coupled model of reservoir and ground pipe network and proposed an implicit method to solve the network node and the reservoir grid. Coats et al. [6] proposed a model of the reservoir/wellbore/surface system integration. The model considered the complex condition of wellbore size and the down-hole equipment and solved the entire system at every step of the Newton iteration. Al-Mutairi et al. [7] calculated the IPR curves by using the pressure in the nearwell drainage area, which overcome the shortcomings of previous sensitivity to the variation of well production when calculating the IPR curves using the grid parameters of reservoirs. Guyaguler et al. [8] proposed a similar approach, but in this method each subdomain needs to be solved repeatedly before reaching equilibrium, and then when the final equilibrium is reached the IPR curve that can reflect the condition of near-well reservoir is obtained. Although this method is time-consuming and the amount of calculation is large, it can reduce the balance error. The IPR curve method is mainly used for the conventional reservoir simulation and the unconventional gas reservoir numerical simulation method is time-consuming. Combined with material balance method for isothermal adsorption of coalbed methane, this paper proposes a method to meet the need of unconventional coalbed methane integrated simulation coupling CBM considering network model, wellbore pressure drop, CBM adsorbed state, and its drainage gas recovery mechanism.

\section{Model Description}

2.1. Wellbore Model. Coal reservoir and surface pipeline network was connected by CBM wellbore. The wellbore flow parameters directly affect gas production and surface network flow state. In the process of CBM production, the production is directly determined by bottom-hole flow pressure (BHFP). Figure 1 shows the annulus fluid distribution in the CBM wellbore. Gas and water enter the surface system from the annulus and tubing, respectively. Fluid in annulus can be distinguished by working fluid level as the gas column in the upper level and aerated fluid column in the lower level. Wellbore annulus pressure drop consists of the pressure drop of both parts. Many researches about calculation of BHFP had been suggested.

2.1.1. Single Phase Flow Model. Cullender and Smith [9] derived the calculation equation for pure gas well bottomhole pressure (BHP) through the analysis of the energy equation for gas steady flow. This equation is known as Cullender-Smith method. Later, Texas Railroad Commission presented another calculation method for pure gas well BHP which is the average temperature mean deviation coefficient method [10]. The equations are as follows:

$$
\begin{aligned}
P_{g} & =\sqrt{P_{c}^{2} e^{2 s}+\frac{1.324 \times 10^{-18} \lambda\left(T Z q_{s c}\right)^{2}}{\left(d_{2}-d_{1}\right)^{3}\left(d_{2}+d_{1}\right)^{2}}\left(e^{2 s}-1\right)}, \\
s & =\frac{0.03418 \gamma_{g} H}{T Z} .
\end{aligned}
$$

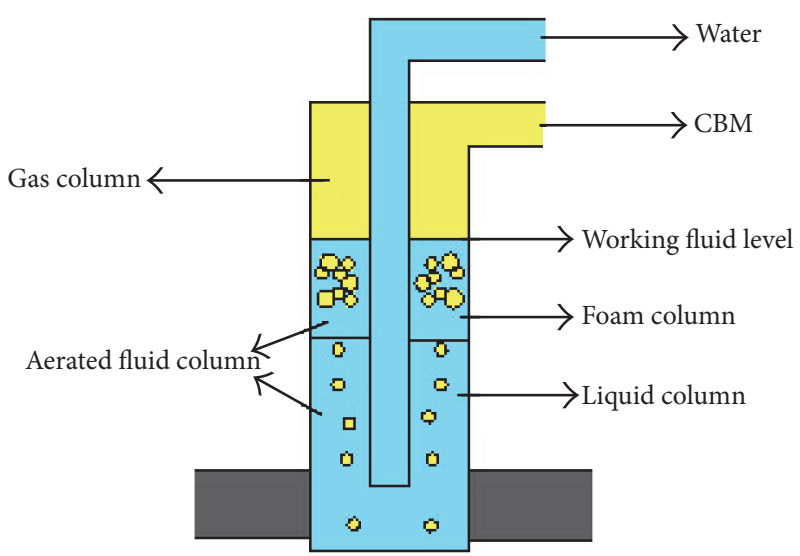

FIgURE 1: The annulus fluid distribution in the CBM wellbore.

2.1.2. Gas-Liquid Phase Flow Model. Takacs and Guffey [11], Chen and Yue [12], Oden and Jennings [13], Hasan and Kabir [14], Liu et al. [15], and Beggs and Brill [16] have proposed different calculation methods, respectively. Among those, Hasan-Kabir's method is as follows:

$$
\begin{aligned}
& P_{w f}=P_{c}+\Delta P_{g}+r_{L} h_{L}-I_{1}+I_{2}, \\
& I_{1}=\frac{C}{a\left(1-f_{g}\right)_{\mathrm{avg}}} \ln \left[1+\frac{a r_{L}\left(1-f_{g}\right)_{\mathrm{avg}} h_{L}}{b C+a\left(P_{c}+\Delta P_{g}\right)}\right], \\
& I_{2}=\frac{M_{g} g C h_{L}}{Z R T a}-\frac{M_{g} g C^{2} b}{Z R a^{2} T r_{L}\left(1-f_{g}\right)_{\mathrm{avg}}} \\
& \cdot \ln \left[1+\frac{a r_{L}\left(1-f_{g}\right)_{\mathrm{avg}} h_{L}}{b C+a\left(P_{c}+\Delta P_{g}\right)}\right], \\
& C=\frac{q_{s c} T Z p_{s c}}{86400 A T_{s c}}, \\
& f_{g}=\frac{v_{s g}}{a+b v_{s g}}, \\
& v_{s g}=\frac{q_{s c} T Z P_{s c}}{A a T_{s c} P} .
\end{aligned}
$$

\subsection{Surface Pipeline Network Model}

2.2.1. Hydraulic Model of Pipe. Steady-state hydraulic calculation for a pipe is used to decide the pipeline pressure drop. Below is the calculation model of gas pipeline pressure drop:

$$
q=\frac{\pi}{4} \sqrt{\frac{\left(P_{\mathrm{Q}}^{2}-P_{Z}^{2}\right) D^{5}}{\lambda Z R T L} .}
$$

2.2.2. Hydraulic Calculation of Pipeline Network. For a pipeline network system with $n$ nodes (wellhead and surface 
system nodes) and $m$ sections, $n$ nodes are corresponding to $n$ flow continuity equations. The node matrix equation formed by those the continuity equations can be written as the following form:

$$
\mathbf{A Q}=\mathbf{q}
$$

Usually, the relationship between the pressure loss and the flow rate of each pipe section could be expressed as the form of a vector function:

$$
\mathbf{Q}=\phi(\Delta \mathbf{P})
$$

Pipe section pressure drop could be expressed by the pressure difference between the two endpoints of the section:

$$
\Delta \mathbf{P}=\mathbf{A}^{T} \mathbf{P} .
$$

Substituting (9), (10), and (11), the mathematical model for the node method could be derived as follows:

$$
\mathbf{A}\left[\phi\left(\mathbf{A}^{T} \mathbf{P}\right)\right]=\mathbf{q} .
$$

2.2.3. Thermodynamic Calculation of Pipeline Network. Steady-state thermodynamic calculation is based on the analysis of steady-state hydraulic analysis. Gas phase temperature drop of the pipeline could be calculated by the Gertjan Zuilhof temperature drop formula which is frequently used in gas pipeline.

$$
T=T_{0}+\left(T_{Q}-T_{0}\right) e^{-a x}
$$

During the solving process, the main aim is to obtain the network node temperature and solve the problem by this parameter. The equation presented by Wei et al. [17] was employed.

$$
T_{i}=\frac{\sum_{k=1}^{m} a_{i k} q_{k} c_{k} T_{k}^{R}+q_{g i} c_{g i} T_{g i}}{q_{i i} c_{i}} .
$$

2.3. Reservoir Model. Three phases, coal, gas and water, coexist in CBM. The unique characteristics of dual porosity system make the productivity prediction different from the method used in conventional gas reservoir. So far, some people tried to predict the production performance using the CBM reservoir numerical simulation [18]. This approach requires a large amount of production data and geological data. It is therefore difficult to solve the model. The long calculation running time limits the application of the numerical method. In this paper, simple but effective material balance method is utilized to forecast the CBM well production performance.

2.3.1. $C B M$ Mining. CBM is mainly stored as an adsorption state on the coal surface. Langmuir sorption isotherm equation is usually used to describe the relationship between the adsorption gas volume and pressure.

$$
V=\frac{V_{L} P_{r}}{P_{L}+P_{r}} .
$$

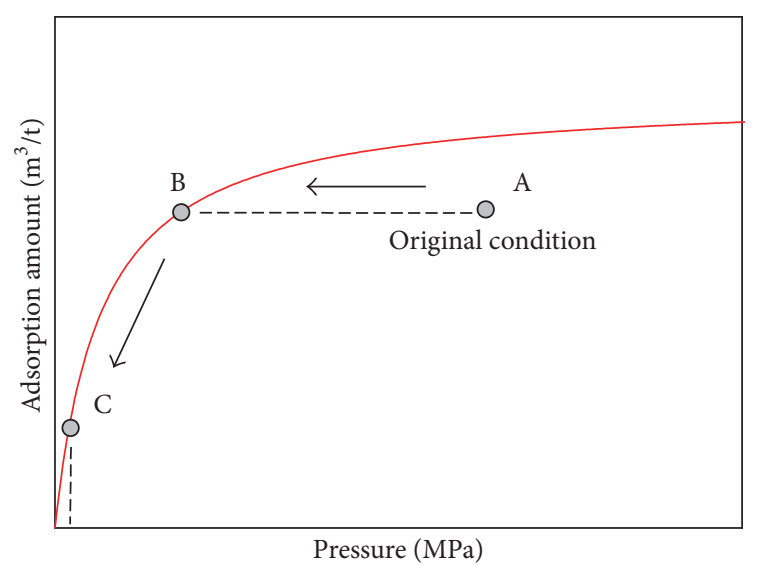

_ Langmuir isothermal adsorption curve

FIGURE 2: Langmuir isothermal adsorption curve.

$P_{r}$ represents the pressure $(\mathrm{MPa}) ; V$ represents the amount of gas at the pressure $P_{r}\left(\mathrm{~m}^{3} /\right.$ ton $) ; V_{L}$ represents the Langmuir volume coefficient $\left(\mathrm{m}^{3} /\right.$ ton); $P_{L}$ represents the Langmuir pressure coefficient $(\mathrm{MPa})$. Langmuir volume coefficient describes the adsorption constant $\left(V_{L}\right)$ of methane adsorption isotherm. The physical meaning of this constant is the adsorbed gas volume when unit quality coal is under saturation condition at a given temperature. Coalbed methane Langmuir pressure coefficient describes the adsorption constants $\left(P_{L}\right)$. The physical meaning of this constant is the pressure when the amount of methane adsorbed on the coal reaches half of the Langmuir volume.

The red curve in Figure 2 shows the Langmuir isothermal adsorption curve when $P_{r}$ is 2.38 and $V_{L}$ is $38.16 \mathrm{~m}^{3} / \mathrm{t}$. The adsorption volume increases with pressure, but when the pressure rises to a certain value, the volume does not change, which means that the adsorption of coal surface is under saturation.

In addition, the Langmuir pressure coefficient is a parameter which affects the shape of isotherm curve of coal adsorption. The smaller the Langmuir pressure coefficient, the greater the degree of bending of the adsorption curve.

Furthermore, adsorption isotherm curve has obvious effect on coalbed methane production. Coalbed can be divided into 3 states [19] in theory, supersaturated, saturated, and undersaturated. In real situation, the coal bed is mostly undersaturated. Figure 2 shows the CBM mining stage in undersaturated condition. Point $\mathrm{A}$ in the picture shows the initial point of the reservoir. Point B is the saturation point. Point $C$ indicates the shut-in pressure. As water exists in coal bed cracks, the coal reservoir pressure can be reduced by pumping the confined water at the beginning till the reservoir pressure reduced to the critical pressure point $B$. Then the adsorbed methane starts releasing a large amount of free methane and flowing to the wellhead. This stage is influenced by coal matrix permeability. As more and more water is discharged, gas production increases rapidly to reach a peak. After the reservoir pressure decreases to a certain level, gas 
production rate will decline gradually $(\mathrm{B}-\mathrm{C})$ until the shut-in condition (C). The whole CBM exploitation cycle completes at this point.

2.3.2. Material Balance Method. The CBM formation reserve equals the sum of the amount of adsorption and free gas.

$$
V_{r}=\rho_{b} A h \frac{V_{L} P_{r}}{P_{L}+P_{r}}+\frac{\phi A h\left(1-S_{w}\right)}{B_{g}} .
$$

Material balance method [20] includes King model, Seidle model, and Jensen-Smith model, in which King model is the most commonly used one. This model assumes that the gas adsorption and desorption equilibrium follow Langmuir sorption isotherms. Gas output can be written as the following form:

$$
G_{p}=\rho_{b} A h V_{L}\left(\frac{P_{i}}{P_{L}+P_{i}}-\frac{P_{r}}{P_{L}+P_{r}}\right) .
$$

Substituting the formation coefficient to (17), the equation can be transformed to the following form:

$$
\begin{aligned}
& G_{p}=\phi_{i} A h \frac{T_{s c} Z_{s c}}{P_{s c} T_{r}}\left(\frac{P_{i}}{Z_{i}^{*}}-\frac{P_{r}}{Z^{*}}\right), \\
& Z^{*} \\
& =\frac{Z}{\left[1-c_{f}\left(P_{i}-P_{r}\right)\right]\left(1-\bar{S}_{W}\right)+\left(\rho_{b} B_{g} / \phi_{i}\right)\left(V_{L} P_{r} /\left(P_{L}+P_{r}\right)\right)}, \\
& \bar{S}_{w}=\frac{S_{w i}\left[1+c_{w}\left(P_{i}-P_{r}\right)\right]+5.615\left(W_{e}-B_{w} W_{P}\right) / \phi_{i} A h}{\left[1-c_{f}\left(P_{i}-P_{r}\right)\right]} .
\end{aligned}
$$

Original gas in place (OGIP) can be calculated as follows:

$$
\text { OGIP }=\phi_{i} A h \frac{T_{s c} Z_{s c} P_{i}}{P_{s c} T_{r} Z_{i}^{*}}
$$

Substituting (21) in (18), a linear relation between the average gas reservoir pressure and the cumulative gas production can be obtained as follows:

$$
\frac{P_{r}}{Z^{*}}=-\frac{P_{i}}{Z_{i}^{*}(\mathrm{OGIP})} G_{P}+\frac{P_{i}}{Z_{i}^{*}} .
$$

At the beginning of undersaturated CBM exploration well, formation water is the main product. Gas production is too small to ignore. Water production in well is constant. The formation pressure difference equation at this time can be written as

$$
\begin{aligned}
P_{r}-P_{w f} & =\frac{q_{w} t}{c_{t} N_{w}}+\frac{141.2 B_{w} \mu_{w} q_{w}}{k h}\left(\ln \frac{r_{e}}{r_{w a}}-\frac{3}{4}\right), \\
N_{w} & =\frac{7758 \phi A h}{B_{w}} .
\end{aligned}
$$

\subsubsection{Productivity Prediction}

(1) Gas Production Equation. Below is the gas production equation for CBM:

$$
q_{g}=\frac{k_{g} h\left[m\left(P_{r}\right)-m\left(P_{w f}\right)\right]}{1422 T\left[\ln \frac{r_{e}}{r_{w}}-\frac{3}{4}+s_{f}\right]} .
$$

Among those, $m(P)$ is the gas pseudo-pressure whose definition is the followed one:

$$
m(P)=\int_{P_{b}}^{P} \frac{P}{\mu_{g} Z} \mathrm{~d} P .
$$

(2) Water Production Equation. Below is the water production equation for CBM:

$$
q_{w}=\frac{k_{w} h\left[P_{r}-P_{w f}\right]}{141.2 \mu_{w} B_{w}\left[\ln \frac{r_{e}}{r_{w}}-\frac{3}{4}+s\right]} .
$$

(3) Relationship between Coal Bed Permeability and Porosity. Coal is composed of cracks and coal matrix. Coal matrix stores gas by adsorption. Diffusion is the primary means of the gas flowing in the matrix. There is a huge difference between the permeability in coal and in conventional fracture gas reservoir. Below is the relationship between the porosity and permeability:

$$
\left(\frac{k_{f}}{k_{0}}\right)=\left(\frac{\phi_{f}}{\phi_{0}}\right)^{n} .
$$

The declination of formation pressure will result in absolute permeability change in the reservoir. This influence can be described using Palmer-Mansoori model [21]:

$$
\begin{aligned}
\frac{\phi}{\phi_{0}}= & 1+\frac{C_{m}}{\phi_{0}}\left(P_{r}-P_{i}\right) \\
& +\frac{\varepsilon_{l}}{\phi_{0}}\left(\frac{K}{M}-1\right)\left(\frac{P_{r}}{P_{L}+P_{r}}-\frac{P_{i}}{P_{L}+P_{i}}\right), \\
C_{m}= & \frac{1}{M}-\left(\frac{K}{M}+f-1\right) \gamma, \\
\frac{K}{M}= & \frac{1}{3}\left(\frac{1+v}{1-v}\right) .
\end{aligned}
$$

With the dehydration of coal, the gas and water in the cracks is in Darcy flow. Coal saturation changes so that the relative gas-water permeability changes as well. So Corey and Rathjens [22] presented the equations below:

$$
\begin{aligned}
\frac{k_{r g}}{k_{r g 0}} & =\left(\frac{S_{g}-S_{g c}}{1-S_{w c}-S_{g c}}\right)^{n_{g}} \quad\left(S_{g}>S_{g c}\right), \\
\frac{k_{r w}}{k_{r w 0}} & =\left(\frac{S_{w}-S_{w c}}{1-S_{w c}}\right)^{n_{w}} \quad\left(S_{g} \geq 1-S_{w c}\right) .
\end{aligned}
$$




\section{Problem Statement}

Coalbed methane production system simulation and deliverability forecasting can be described below. The following parameters are given:

(1) reservoir parameters: initial reservoir pressure, reservoir temperature, coalbed thickness, and so on,

(2) basic wellbore parameters: tubing diameter, inner diameter, well depth, liquid level depth, drilling fluid density, and so on,

(3) surface pipeline network: network structure, pipe diameter, and so on,

(4) composition of CBM.

The following parameters need to be determined:

(1) reservoir pressure,

(2) bottom hole flowing pressure,

(3) gas rate,

(4) water rate,

(5) node pressure and flow rate of the pipeline network.

\section{Solution Algorithm}

4.1. Calculation Algorithm of BHFP. The calculation process of BHFP is described as follows:

(1) The pressure of the working fluid level $P_{g}$ is unknown. To obtain the average pressure and average temperature, we should first assume the initial value of $P_{g}$.

(2) The gas deviation factor and the friction coefficient at the average pressure and average temperature will be then calculated.

(3) Substitute the results in (1) to calculate $P_{g}$.

(4) Comparing the calculated result and the assumed value of $P_{g}$, if the difference of $P_{g}$ does not meet the error requirement, the calculated $P_{g}$ will be used as the assumed value. Then repeat step (1) to step (3) until the difference of $P_{g}$ meets the error requirement.

(5) The initial value of BHFP $P_{w f}$ should also be assumed. The average pressure and average temperature will be calculated then.

(6) The average deviation coefficient $Z$ could be calculated based on the average pressure and average temperature.

(7) According to (7), $v_{s g}$ can be calculated to determine the value of $a$ and $b$.

(8) After evaluating $I_{1}$ and $I_{2}, P_{w f}$ can be calculated from (2).

(9) Comparing the calculated result and the assumed value of $P_{w f}$, if the difference of $P_{w f}$ does not meet the error requirement, the calculated $P_{w f}$ will be used as the assumed value. Then repeat step (5) to step (8) until the difference of $P_{w f}$ meets the error requirement.
4.2. Surface Network Parameters Calculation. During the calculation process of gas phase pipeline network, the hydraulic calculation and thermodynamic calculation influence each other; therefore, the entire calculation is a coupling hydraulic/thermodynamic iterative process. The specific calculation steps are described below:

(1) Input basic data of the pipeline network, including pipe length, diameter, absolute roughness, gas composition, ambient temperature, and overall heat transfer coefficient.

(2) The initial value of node pressure vector $\mathbf{P}_{0}$, node flow vector $\mathbf{q}_{0}$ and node temperature vector $\mathbf{T}_{0}$ should be assumed. The initial value of $k$ is 1 .

(3) The solution (12) should be calculated using the node method for steady-state hydraulic pipe network. The node pressure vector $\mathbf{P}_{k}$ and node flow vector $\mathbf{q}_{k}$ under the current node temperature vector $\mathbf{T}_{k-1}$ could be both obtained.

(4) According to (13), the temperature drop vector $\Delta \mathbf{T}_{k}$ under $\mathbf{P}_{k}$ and $\mathbf{q}_{k}$ for each pipe branch can be calculated.

(5) The solving sequence of the network node temperature should be established.

(6) Node temperature vector $\mathbf{T}_{k}$ can be solved by temperature for each node calculated from the solving sequence and (14).

(7) If $\left|\mathbf{T}_{k}-\mathbf{T}_{k-1}\right|<\varepsilon$ ( $\varepsilon$ is the error precision), the calculation can be stopped. If not, $\mathbf{T}_{k}$ should be treated as the initial node temperature vector for a new iterative calculation circle, and $k=k+1$. Then repeat step (3) to step (7).

4.3. Reservoir Simulation. Coal reservoir production can be roughly predicted if the material balance equation and the CBM gas/water production equation are combined with the known BHFP. The specific steps are as follows:

(1) Input basic data of reservoir, including Langmuir volume, Langmuir pressure, bulk density, initial reservoir pressure, and porosity.

(2) OGIP can be obtained by (21). Then the desorption pressure corresponding with the gas reserves can be obtained. This result will be compared to the gas reservoir pressure at this time.

(3) If gas reservoir pressure is bigger than desorption pressure, that means the coalbed is undersaturated. Water production rate at this time $q_{w}$ and the cumulative water production in a period $\Delta \mathrm{T}$ can be calculated by (26). Then this cumulative water production can be utilized to calculate the gas reservoir pressure at the end of the time period. Repeat step (3) until the gas reservoir pressure equals the desorption pressure. Then proceed to step (4).

(4) If gas reservoir pressure equals the desorption pressure (supersaturated state of the coal is not considered 


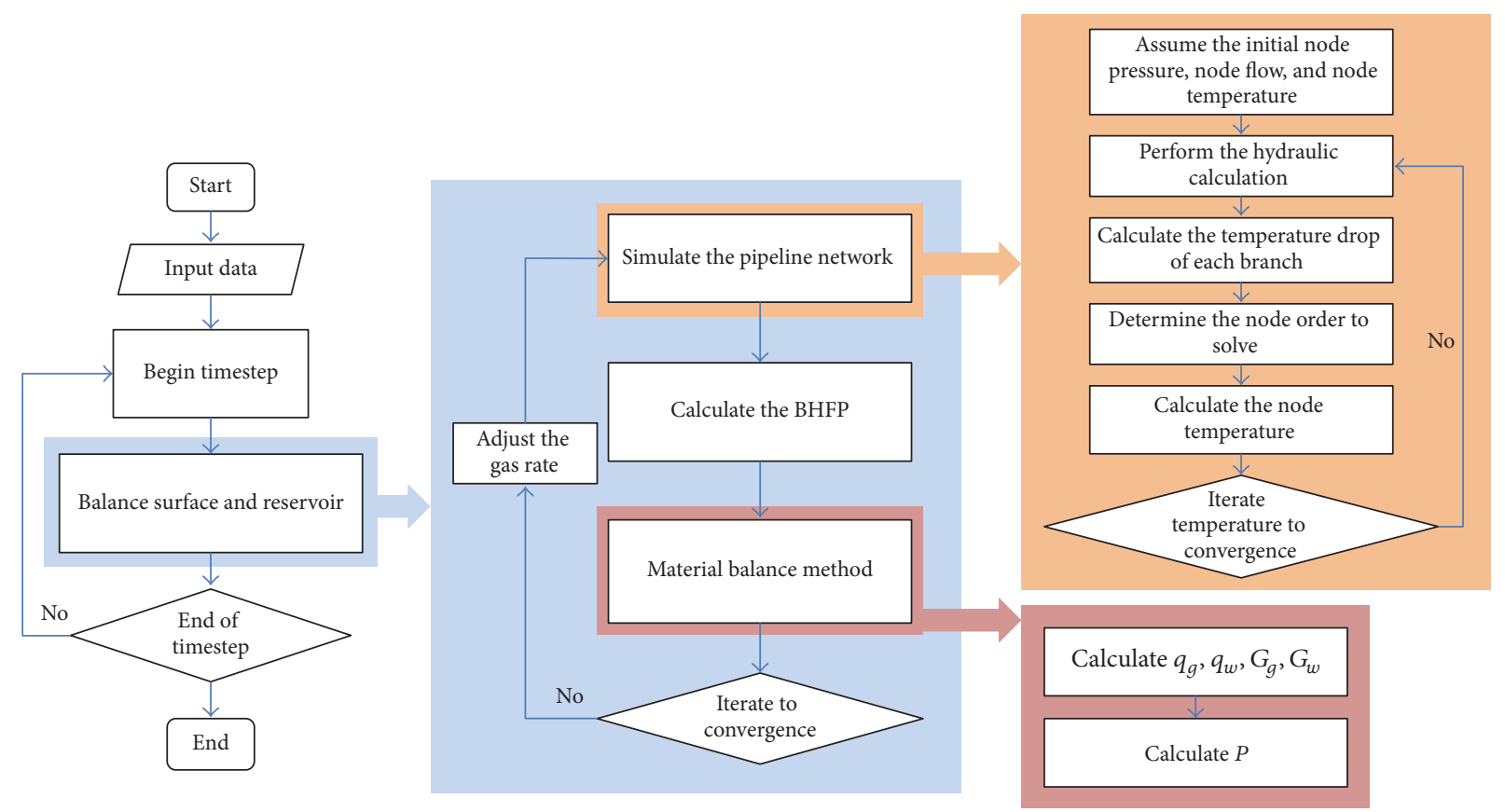

FIGURE 3: CBM reservoir/surface coupling algorithm.

here), that means the coalbed is saturated. Both gas and water will be produced from the coalbed. $Q_{w}$ will be calculated. The gas production per unit time $q_{g}$ and the cumulative gas production and cumulative water production can be calculated from (24). Then the gas reservoir pressure at the end of the time period can be calculated. Repeat step (4) until it reaches the shut-in pressure.

4.4. Production System Coupling Calculation. The basic assumptions of CBM production system coupling calculation are as follows:

(1) During the gas production process of CBM, although the gas production changes with time, it still can be treated as constant in a small time interval. In this time interval, the flow in the wellbore and the surface pipe network can be regarded as a steady flow.

(2) In the actual production, the working liquid level in the wellbore always changes due to the influence of gas production, water production, and the formation condition. The main factor is the production rate. In this case, the working liquid level is assumed constant.

Figure 3 shows the flow chart of CBM production system coupling calculation.

CBM production system coupling calculation model is the unity of CBM well productivity prediction model, wellbore calculation model, and surface pipe network model. The production indexes such as formation pressure, bottom hole pressure, and gas production can be determined by coupling iterations of the three models. This calculation model can be employed to optimizing the production plan. The specific calculation process is described below:

(1) Input the basic data of CBM reservoir, wellbore, and surface network.

(2) Do the surface, wellbore, and reservoir coupling calculation.

(3) Assume the initial iteration value of gas production for each well at this time; then calculate the wellhead pressure for each well according to the surface pipe network model.

(4) According to the calculated initial value of wellhead pressure and gas production, calculate the BHFP for each well using the wellbore model, respectively.

(5) According to the calculated BHFP, calculate the gas production at the end of the production period for each well using the CBM reservoir productivity prediction model.

(6) Compare the calculated value and the assumed value. If the difference satisfies the requirements of the error precision, calculate the cumulative gas production and cumulative water production. If not, replace the calculated value as the initial iteration value and then repeat step (3) to step (5).

(7) See whether it reaches the end of the production period or not. If yes, the calculation ends. If not, repeat step (2) to step (5).

\section{Examples}

5.1. Evaluation of BHFP Calculation Method. In the calculation of CBM BHFP, wellhead casing pressure data can be 
generally read by the wellhead pressure gauge. The pressure difference of pure gas column and the pressure difference of mixed gas liquid column can be calculated from the model introduced above. The sum of these three values is the BHFP. Although many scholars have proposed different methods to calculate BHFP, they did not compare or evaluate the applicable range and calculation accuracy.

In this paper, different calculation models have been studied and effective model with higher calculation accuracy is recommended by comparing different models. Study shows that the results of average temperature, average deviation coefficient method, and the results of Cullender-Smith method are approximately the same [10]. So the average temperature average deviation coefficient method is used to calculate the pressure difference for pure gas column. The following four models to calculate CBM BHFP are studied by combining the method for calculating mixed gas liquid pressure difference.

Model 1. Average temperature and average deviation coefficient method is used to calculate the pressure difference for pure gas column. Jialang Chen-Xiang'an Yue method [12] is used to calculate the pressure difference for mixed gas liquid column.

Model 2. Average temperature and average deviation coefficient method is used to calculate the pressure difference of pure gas column. Hasan-Kabir analytic method [14] is used to calculate the pressure difference for mixed gas liquid column.

Model 3. Average temperature and average deviation coefficient method is used to calculate the pressure difference for pure gas column. Beggs-Brill method [16] is used to calculate the pressure difference for mixed gas liquid column.

Model 4: Xinfu Liu Method. To obtain the optimized model to calculate CBM BHFP, these 4 models are used for 21 gas wells and the results will be compared with the field measured data. In Figure 4, the red line shows the field measured data of BHFP. Data number 1 to number 6 (dataset 1) are from [23], data number 7 to number 15 (dataset 2) are from [15], and data number 16 to number 21 (dataset 3 ) are the measured data from a certain block of field. Figures 4 and 5 are the calculation results and the relative error of each model.

After comparing these 4 models, the result of Model 1 for dataset 2 is close to the measured value, yet the calculation result error is large, which means the calculation precision of this model changes with the gas well conditions. The same result can be drawn from Model 3 as well. The calculation results of Model 1 for dataset 1 and dataset 3 are both close to the measured result. Using Model 2, we can also obtain the result close to the measured value. The error is within $20 \%$ and calculation accuracy is relatively high.

Table 1 is the summary of the application, calculation accuracy, and the advantages and disadvantages of each model. From the present result, though the calculation results of Jialang Chen-Xiang'an Yue method and HasanKabir analytic method are close to each other, Jialang ChenXiang'an Yue method has a narrower applicable condition

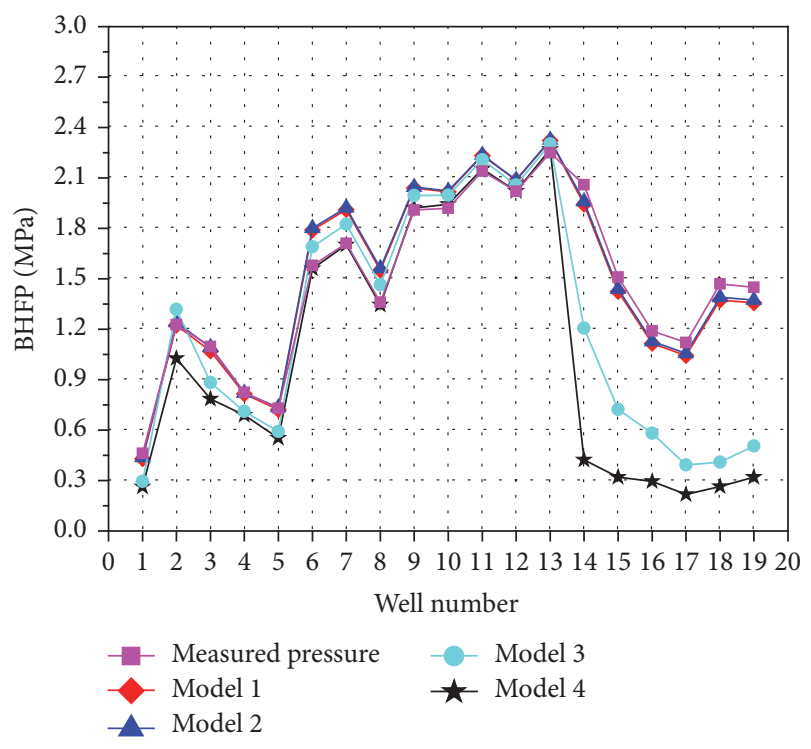

FIGURE 4: Comparison of calculated result with measured value.

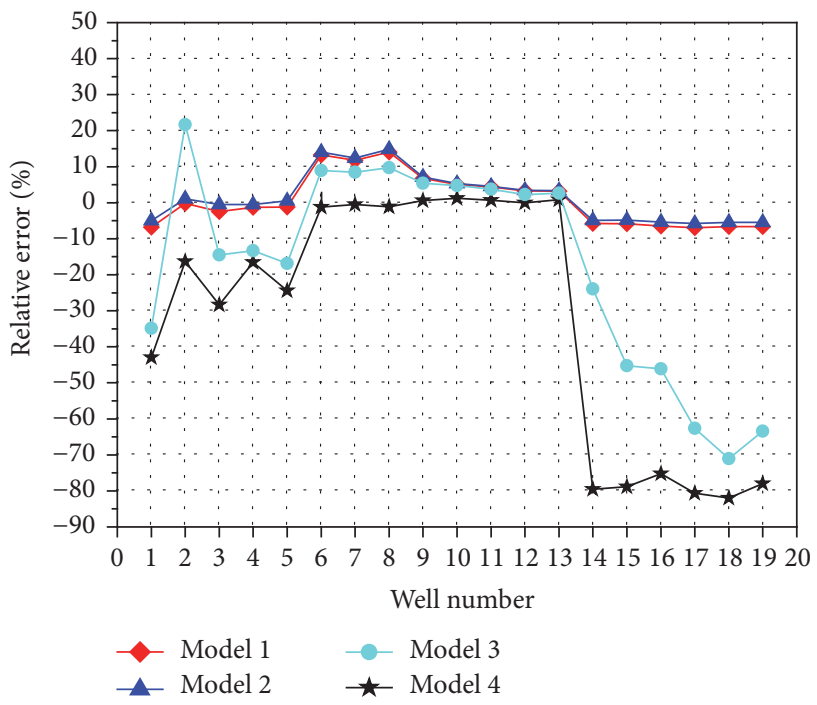

FIgURE 5: Relative errors of calculated result.

which is GCF $>0.3$. Among the 3 models, Hasan-Kabir analytic method has a relatively high calculation accuracy and wide applicability. So this model is chosen to calculate the CBM well BHFP.

5.2. Example 1. Coupled calculation method was applied to 2 blocks of a CBM field. System structure is illustrated in Figure 6, which is a star shaped structure. The output for each well will be collected to the center node (Node 12) through a separate line.

Coupling algorithms are used for productivity prediction. The parameters of coal reservoir and gas composition are given in Tables 2 and 3.

(1) Daily Gas Production. Predicted gas production of each well is shown in Figure 7. 


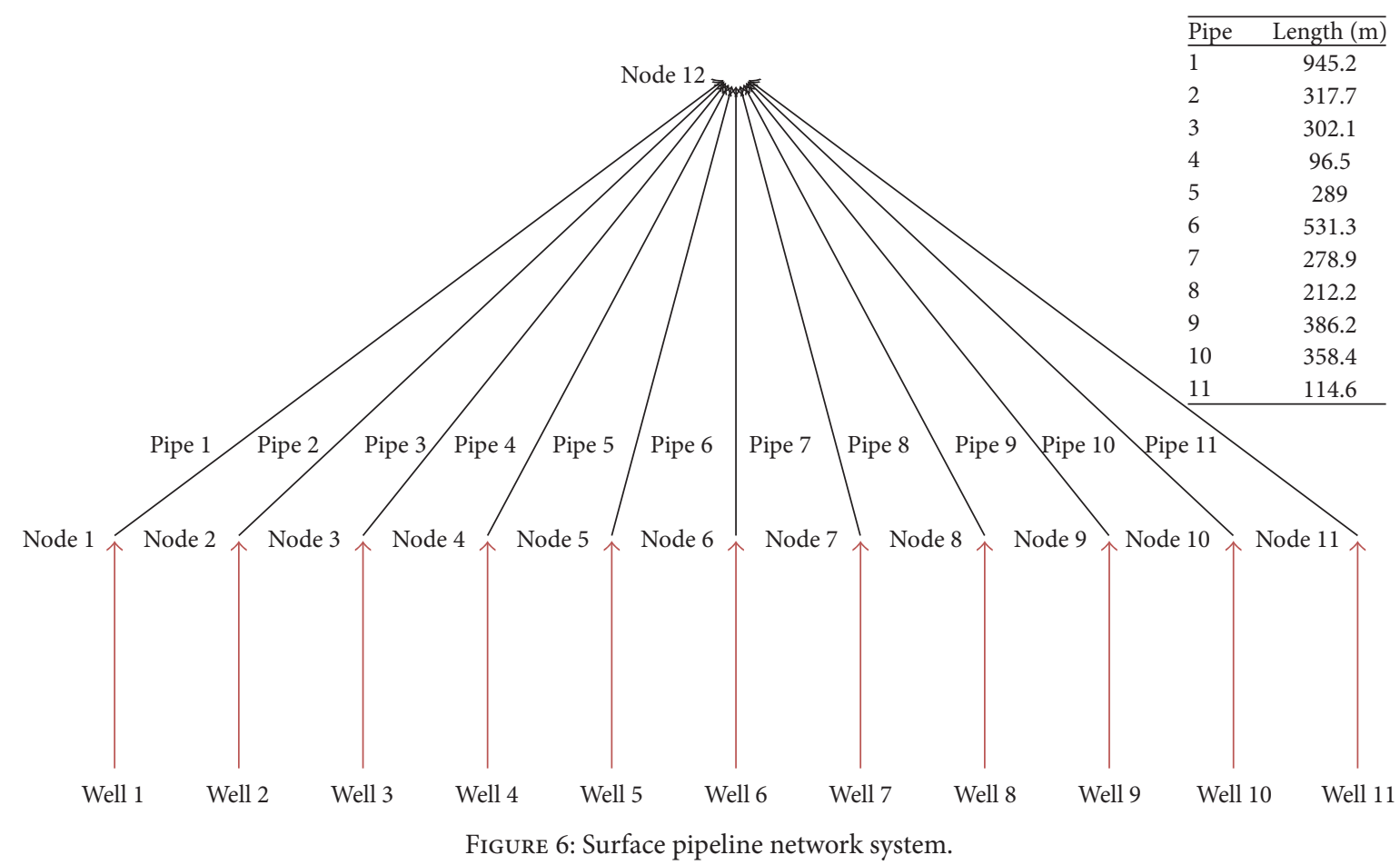

TABLE 1: Comparison of the four models.

\begin{tabular}{llll}
\hline Calculation method & Application & Calculation accuracy & Advantages/disadvantages \\
\hline Model 1 & GCF $>0.3$ & Relatively high & $\begin{array}{l}\text { High precision, but large amount of calculation, narrow } \\
\text { application scope } \\
\text { Model } 2\end{array}$ \\
All cases & Relatively high & $\begin{array}{l}\text { Simple calculation process, high precision, good } \\
\text { stability }\end{array}$ \\
Model 3 & All cases & Change with the gas well conditions & $\begin{array}{l}\text { Complex calculation process, poor stability } \\
\text { Model } 4\end{array}$ \\
\hline
\end{tabular}

TABLE 2: Parameters of coal reservoir.

\begin{tabular}{lc}
\hline Input parameters & Value \\
\hline Initial reservoir pressure $(\mathrm{MPa})$ & 5.28 \\
Reservoir temperature $(\mathrm{K})$ & 304.15 \\
Initial porosity $(\%)$ & 4.5 \\
Formation thickness $(\mathrm{m})$ & 6.2 \\
Drainage area $\left(\mathrm{m}^{2}\right)$ & 90000 \\
Bulk density $\left(\mathrm{t} / \mathrm{m}^{3}\right)$ & 1.45 \\
Gas content $\left(\mathrm{m}^{3} / \mathrm{t}\right)$ & 14.1 \\
Langmuir volume $\left(\mathrm{m}^{3} / \mathrm{t}\right)$ & 38.16 \\
Langmuir pressure $(\mathrm{MPa})$ & 2.38 \\
\hline
\end{tabular}

TABle 3: Composition of CBM.

\begin{tabular}{lcccc}
\hline Composition & $\mathrm{CH}_{4}$ & $\mathrm{C}_{2} \mathrm{H}_{6}$ & $\mathrm{~N}_{2}$ & $\mathrm{CO}_{2}$ \\
\hline Mole present (\%) & 96.17 & 0.05 & 3.71 & 0.07 \\
\hline
\end{tabular}

Figure 7 shows the gas production for each well in the next 10 years. As can be seen from Figure 7, the daily gas production change trend of all wells is basically identical. In the initial production stage, water is the main product. Gas production is 0 . As time goes by, these 11 gas wells begin to produce gas. The gas production of each well is close to each other, and they increase year by year at the beginning and then decrease afterwards. The peak appears in the 2280 th days at about $2800 \mathrm{~m}^{3} / \mathrm{d}$.

(2) Daily Gas Production. Water production of each gas well under the star shaped gathering structure is shown in Figure 8.

Figure 8 shows the water production for each well in the next 10 years. As can be seen from Figure 8, the daily water production is nearly the same with obvious change trend. In the initial time of production, all gas wells begin to show formation water and the production rate is $35.67 \mathrm{~m}^{3} / \mathrm{d}$. Along with the water emergence, the formation pressure decreased gradually to the critical desorption pressure of CBM. Gas begins to desorb. Throughout the whole gas production process, formation water discharged from each gas well reduces gradually. In the 10 th year, it reaches $1.97 \mathrm{~m}^{3} / \mathrm{d}$. In the later stages of production, water production of each gas well 


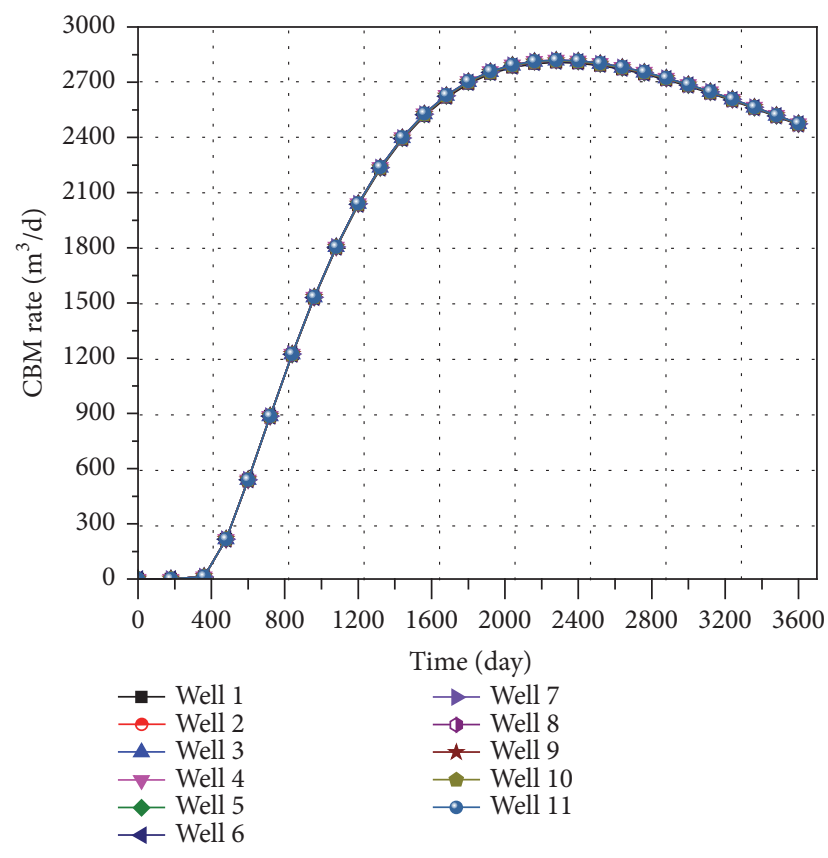

Figure 7: Daily well gas productions.

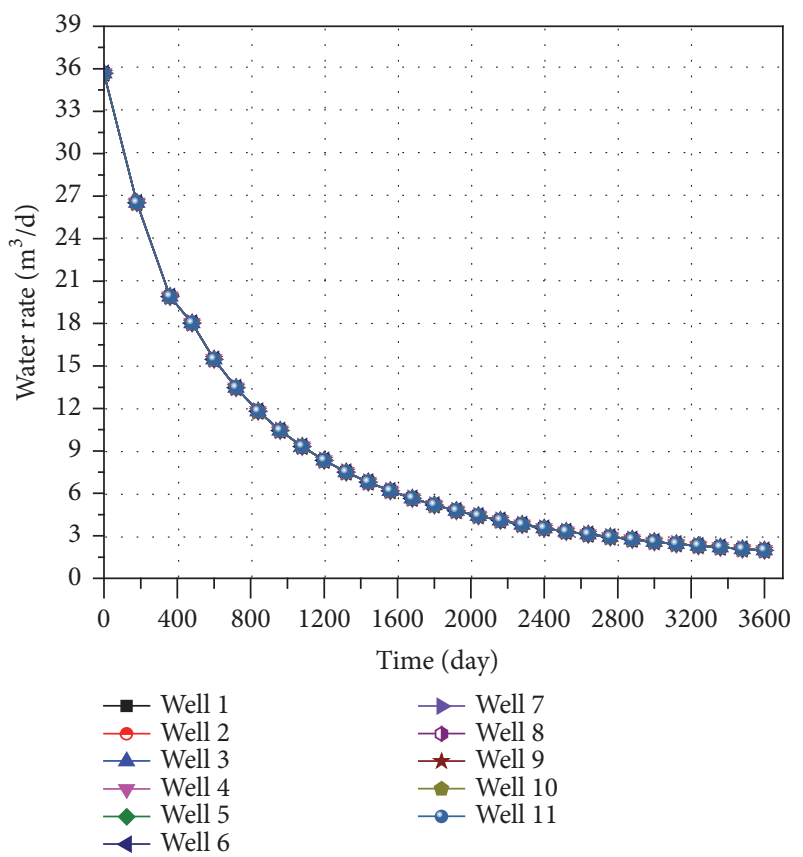

FIGURE 8: Daily well water productions.

become less and less and almost no water is produced after a period of time.

(3) Formation Pressure. The formation pressure changes are shown in Figure 9.

Figure 9 is the reservoir pressure of each well with the change of time. As can be seen from Figure 9, the gas reservoir pressure of each well is almost the same which all decreases with the time. During this process, water production of

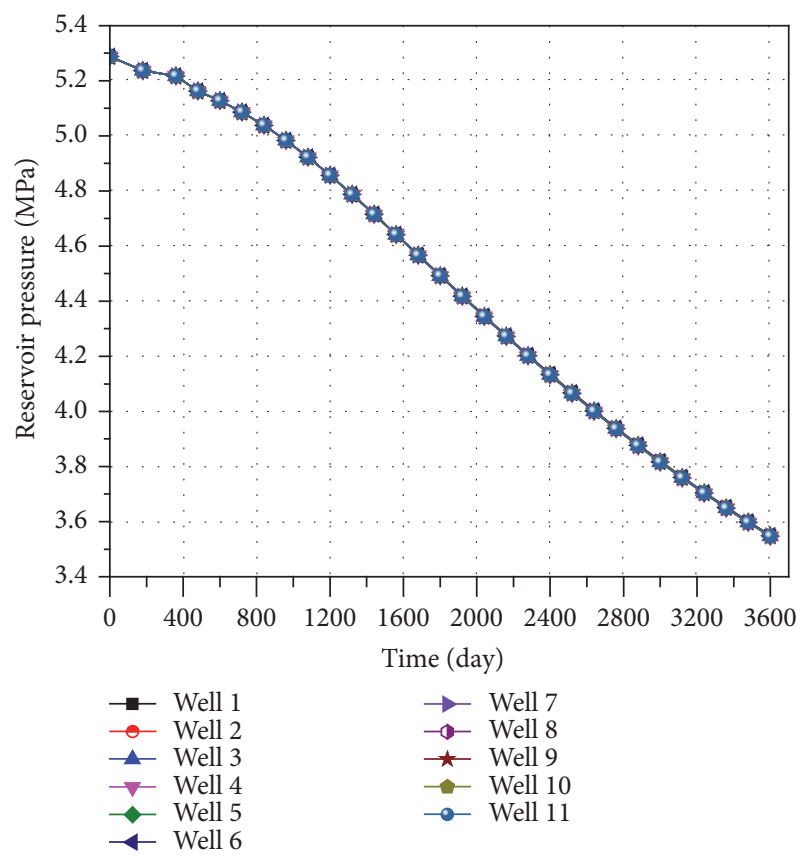

FIgURE 9: Reservoir pressures.

each gas well reduces gradually. When the reservoir pressure decreases to the critical desorption pressure of CBM, gas begins to desorb from the surface of the coal matrix and comes out from the wellhead. In the 10th year after production, reservoir pressure drops from 5.28 MPa to $3.55 \mathrm{~Pa}$.

5.3. Example 2. System structure is shown in Figure 10, which is a dendritic surface system.

(1) Daily Gas Production. Future gas production for each gas well is shown in Figure 11.

Figure 11 shows the gas production for each well in the next 10 years. It can be observed in Figure 11 that the daily gas production for each well is approximately the same at the beginning of production but different significantly afterwards. The largest production goes to Well 7 which is the nearest to the collection point (Node 12), while the smallest production is of Well 1 which is the farthest to the collection point. However, during the whole process, the change trend of gas production for each well is consistent basically. At the first time, only formation water is desorbed so the gas production is 0 . As time goes by, these 11 gas wells begin to produce with the gas production increasing at the beginning and decreasing after a few years. Nevertheless, the peak time for each well is not the same. Among them, the gas production of Well 1, Well 2, Well 3, and Well 4 arrives to the peak in the 2520th day, while the gas production of Well 5 and Well 11 reaches a peak in the 2400 th day.

(2) Daily Water Production. Future water production for each gas well is shown in Figure 12.

Figure 12 is the water production for each well in the next 10 years. As shown, the water production for each well 


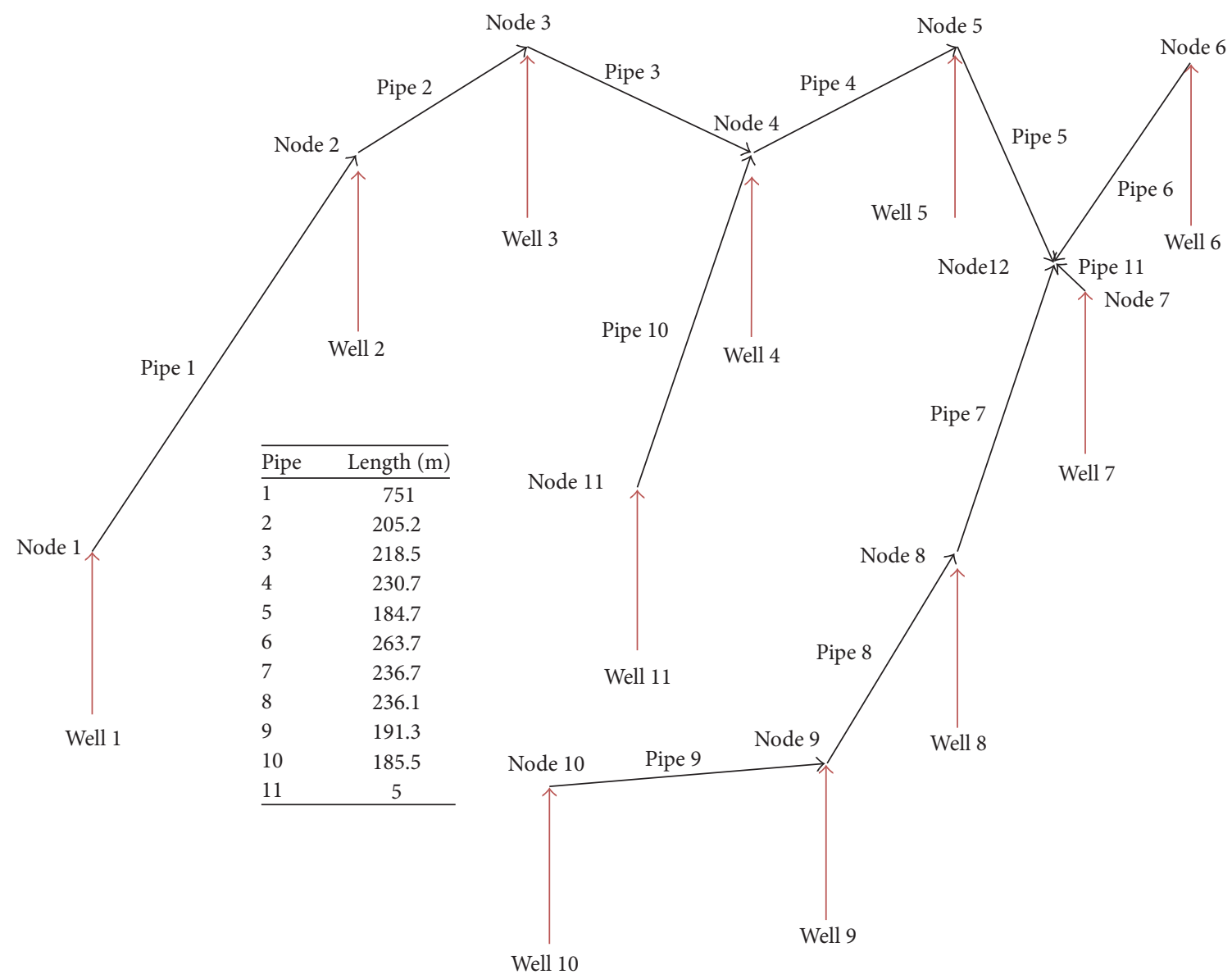

FIGURE 10: Surface pipeline network system.

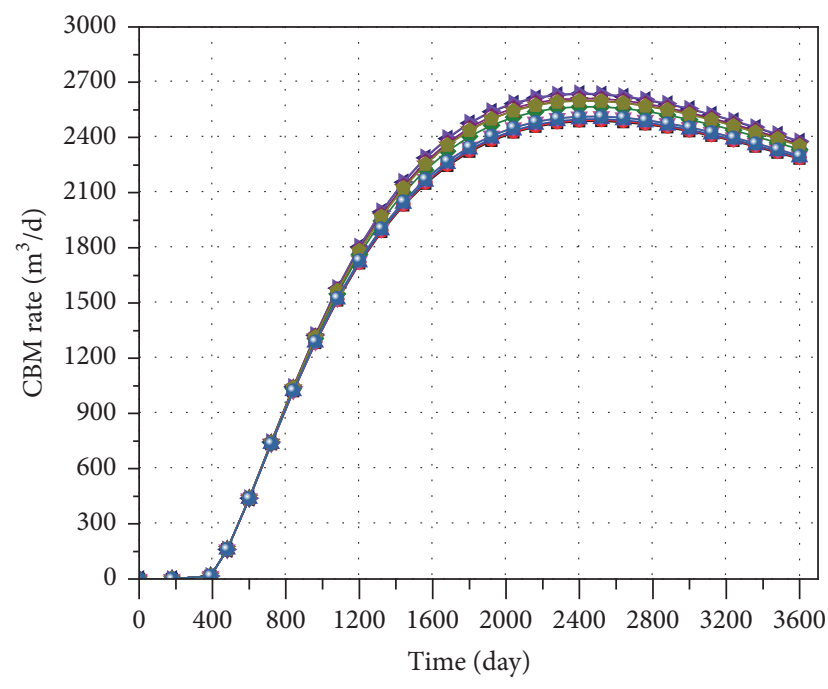

- Well 1

- Well 2

- Well 3

$\rightarrow$ Well 4

Well 5

- Well 6

FIGURE 11: Daily well gas productions.
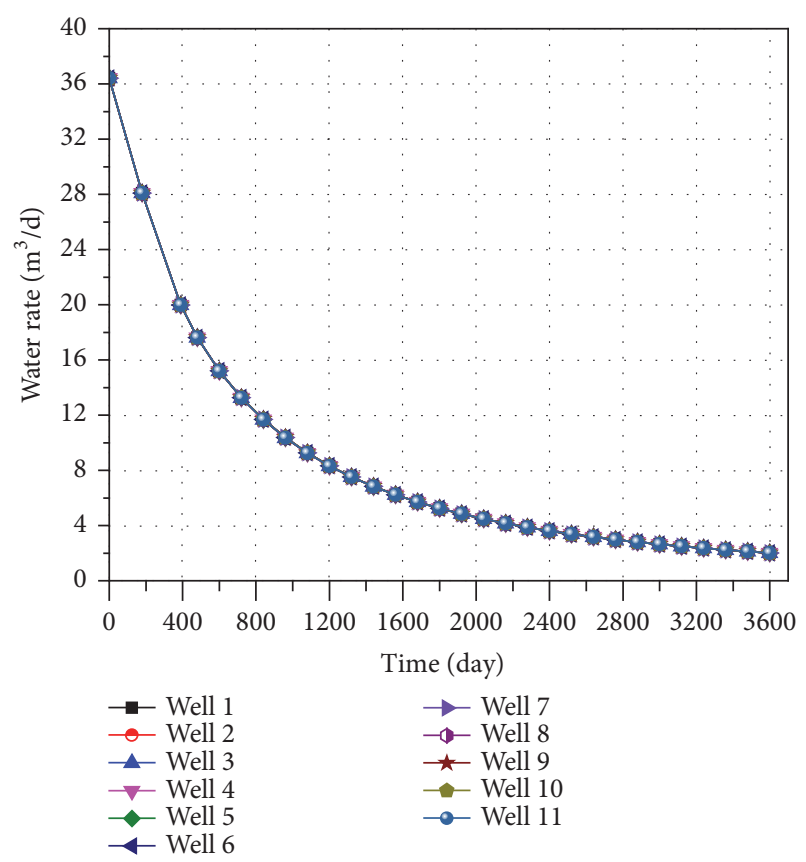

FIGURE 12: Daily well water productions. 


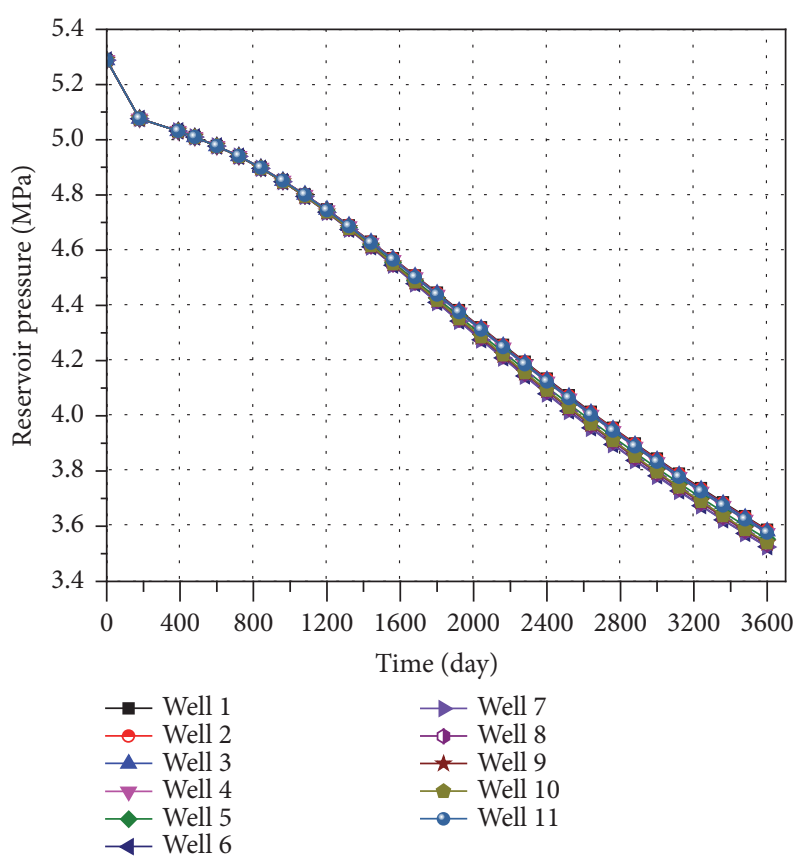

FIGURE 13: Reservoir pressures.

is basically the same. At the beginning of production, the formation water begins to desorb from formation with the production $36.41 \mathrm{~m}^{3} / \mathrm{d}$ for each well. Along with the large amount of water abjection, the reservoir pressure reduces gradually to the critical desorption pressure and then gas begins to desorb. During the entire gas production, water production for gas well decreases gradually to $2.02 \mathrm{~m}^{3} / \mathrm{d}$ in the 10th year. In the late stage of production, water production keeps on decreasing and almost no more water is produced after a period of time.

(3) Formation Pressure. The formation pressure changes are illustrated in Figure 13.

Figure 13 is the reservoir pressure for each well with the change of time. At the beginning of production, the reservoir pressure of each well is nearly the same. Later, they are slightly different from each other. The largest reservoir pressure goes to Well 7 which is the nearest to the collection point (Node 12), while the smallest reservoir pressure is Well 1 which is the farthest to the collection point. During the whole production process, reservoir pressure for each well decreases with different decline rate at different periods. In the initial production stage, the decline rate of reservoir pressure is fast and then the pressure falls more slowly. In the 10th year, reservoir pressure falls to $3.53 \mathrm{MPa}$ from original 5.28 $\mathrm{MPa}$.

\section{Summary}

This paper describes a coupling surface/wellbore/reservoir simulation algorithm which can be used to predict gas production and water production for a period of time. Node method is used for the surface system simulation. Thermodynamic and hydraulic calculation are coupled together to calculate. CBM BHFP shows that the combination of HasanKabir analytic method and average temperature average deviation coefficient method can provide a relatively high accuracy. The advantages and disadvantages of different combination models are listed as well. CBM productivity prediction is based on material balance. The method presented in this paper can be used to assist the CBM system analysis for CBM engineers by 2 validation examples.

\section{Nomenclature}

a: Coefficient, dimensionless

$a_{i k}$ : Element of $\mathbf{A}$

A: Drainage area, $\mathrm{m}^{2}$

$A_{a}$ : Sectional area of annulus, $\mathrm{m}^{2}$

A: Correlating matrix of the node and pipe

$\mathbf{A}^{\mathrm{T}}$ : Transpose matrix of $A$

$b$ : Coefficient, dimensionless

$B_{g}$ : Gas formation volume factor, $\mathrm{m}^{3} / \mathrm{Nm}^{3}$

$B_{w}$ : Water formation factor, $\mathrm{m}^{3} / \mathrm{Nm}^{3}$

$c_{f}$ : Formation compressibility, $\mathrm{MPa}^{-1}$

$c_{i}$ : Heat capacity of the medium which flows out from node $i, \mathrm{~J} /(\mathrm{kg} \cdot \mathrm{K})$

$c_{k}$ : Heat capacity of the medium in section $k$, $\mathrm{J} /(\mathrm{kg} \cdot \mathrm{K})$

$c_{g i}$ : Heat capacity of the medium which flows into the network from node $i, \mathrm{~J} /(\mathrm{kg} \cdot \mathrm{K})$

$c_{t}: \quad$ Total compressibility, $\mathrm{MPa}^{-1}$

$c_{w}$ : Water compressibility, $\mathrm{MPa}^{-1}$

$C_{m}$ : Matrix compressibility, $\mathrm{MPa}^{-1}$

$d_{1}$ : Tubing outside diameter, $\mathrm{m}$

$d_{2}$ : Tubing inside diameter, $\mathrm{m}$

$d h_{L}$ : Step length of aerated fluid column, m

$D:$ Internal diameter, $\mathrm{m}$

e: Absolute roughness, $\mathrm{m}$

$f$ : Tuning factor, dimensionless

$f_{g}$ : Gas porosity, dimensionless

$g: \quad$ Gravity acceleration, $\mathrm{m} / \mathrm{s}^{2}$

$G_{p}:$ Produced gas, $\mathrm{m}^{3}$

$h$ : Formation thickness, $\mathrm{m}$

$h_{L}$ : Aerated fluid column length, $\mathrm{m}$

$H$ : $\quad$ Gas column length, $\mathrm{m}$

$k$ : $\quad$ Permeability, md

$k_{0}$ : Initial permeability, md

$k_{f}$ : Final permeability, md

$k_{g}$ : Effective permeability to gas, md

$k_{w}$ : $\quad$ Effective permeability to water, $\mathrm{md}$

$k_{r g}$ : Relative permeability to gas, dimensionless

$k_{r w}$ : Relative permeability to water, dimensionless

$k_{r g 0}$ : Final relative permeability to gas, dimensionless

$k_{r w 0}$ : Final relative permeability to water, dimensionless

$K$ : $\quad$ Bulk elastic modulus, $\mathrm{MPa}$

L: $\quad$ Pipe length, $\mathrm{m}$

M: Axial constraint modulus, $\mathrm{MPa}$ 
$M_{g}:$ Gas molar mass, $\mathrm{kg} / \mathrm{mol}$

$n$ : Exponent, dimensionless

$n_{g}$ : Exponential of relative gas permeability curve, dimensionless

$n_{w}$ : Exponential of relative water permeability curve, dimensionless

$N_{w}$ : Original water in place, $\mathrm{m}^{3}$

$P$ : $\quad$ Average pressure, $\mathrm{MPa}$

$P_{b}$ : Arbitrary base pressure, $\mathrm{MPa}$

$P_{c}$ : $\quad$ Surface casing pressure, $\mathrm{MPa}$

$P_{g}$ : Pressure at working fluid level, $\mathrm{MPa}$

$P_{i}$ : Initial reservoir pressure, $\mathrm{MPa}$

$P_{L}$ : Langmuir pressure, $\mathrm{MPa}$

$P_{\mathrm{Q}}$ : Inlet pressure of pipe, $\mathrm{MPa}$

$P_{r}$ : Average reservoir pressure, $\mathrm{MPa}$

$P_{Z}$ : Outlet pressure of pipe, $\mathrm{MPa}$

$P_{w f}$ : Bottom-hole flowing pressure, $\mathrm{MPa}$

$P_{s c}$ : Standard pressure, $\mathrm{MPa}$

$\Delta P_{g}$ : Pressure drop of gas column, $\mathrm{MPa}$

P: $\quad$ Node pressure vector

$\Delta \mathbf{P}$ : Pipe pressure drop vector

q: $\quad$ Mass flow rate, $\mathrm{kg} / \mathrm{s}$

$q_{g}: \quad$ Gas rate, $\mathrm{m}^{3} / \mathrm{d}$

$q_{k}$ : Mass flow of the medium in section $k, \mathrm{~kg} / \mathrm{s}$

$q_{w}:$ Water rate, $\mathrm{m}^{3} / \mathrm{d}$

$q_{g i}$ : Mass flow of the medium which flows into the network from node $i, \mathrm{~kg} / \mathrm{s}$

$q_{i i}$ : Total mass flow of the medium which flows into node $I, \mathrm{~kg} / \mathrm{s}$

$q_{s c}$ : Gas production rate in standard state, $\mathrm{m}^{3} / \mathrm{d}$

q: Node flow vector

Q: Pipe flow vector

$r_{e}:$ External radius of reservoir, $\mathrm{m}$

$r_{L}: \quad$ Liquid gravity, $\mathrm{Pa} \cdot \mathrm{m}^{-1}$

$r_{w}$ : Wellbore radius, $\mathrm{m}$

$r_{w a}$ : Apparent wellbore radius, $\mathrm{m}$

$R: \quad$ Universal gas constant, $\mathrm{J} /(\mathrm{mol} \cdot \mathrm{K})$

$s:$ Definition parameter, dimensionless

$s_{f}$ : Skin factor, dimensionless

$S_{g}$ : Average gas saturation, dimensionless

$S_{w}$ : Water saturation, dimensionless

$S_{g c}$ : Irreducible gas saturation, dimensionless

$S_{w c}$ : Irreducible water saturation, dimensionless

$S_{w i}$ : Initial water saturation, dimensionless

$\bar{S}_{w}$ : Average water saturation, dimensionless

$T: \quad$ Temperature, $\mathrm{K}$

$T_{0}$ : Ambient temperature, $\mathrm{K}$

$T_{i}$ : $\quad$ Temperature of node $i, \mathrm{~K}$

$T_{r}$ : Reservoir temperature, $\mathrm{K}$

$T_{Q}$ : Temperature of the starting point of the pipeline, $\mathrm{K}$

$T_{g i}$ : Temperature of the medium which flows into the network from node $i, \mathrm{~K}$

$T_{s c}$ : Standard temperature, $\mathrm{K}$

$T_{k}^{R}$ : Temperature of the end of section $k, \mathrm{~K}$

$V_{s g}:$ Apparent velocity, $\mathrm{m} / \mathrm{s}$
$V: \quad$ Gas content, $\mathrm{m}^{3} / \mathrm{t}$

$V_{L}:$ Langmuir volume, $\mathrm{m}^{3} / \mathrm{t}$

$V_{r}:$ Reserve volume, $\mathrm{m}^{3}$

$W_{e}$ : Encroached water, $\mathrm{m}^{3}$

$W_{p}:$ Produced water, $\mathrm{m}^{3}$

$Z$ : Gas compressibility factor, dimensionless

$Z_{s c}$ : Standard gas compressibility factor, dimensionless

$Z^{*}$ : Gas factor for unconventional gas reservoir, dimensionless

$v$ : Poisson ratio, dimensionless

$\mu_{g}:$ Gas viscosity, Pa.s

$\mu_{w}$ : Water viscosity, Pa.s

$\varphi$ : Porosity, dimensionless

$\varphi_{0}$ : Initial porosity, dimensionless

$\varphi_{f}$ : Final porosity, dimensionless

$\varepsilon_{l}$ : Maximum strain, dimensionless

$\gamma$ : Matrix shrinkability, $\mathrm{MPa}^{-1}$

$\gamma_{g}$ : Gas relative density, dimensionless

$\lambda$ : Hydraulic friction coefficient, dimensionless

$\rho_{b}$ : Bulk density of the coal, $\mathrm{t} / \mathrm{m}^{3}$.

\section{Competing Interests}

The authors declare that there is no conflict of interests regarding the publication of this paper.

\section{Acknowledgments}

The authors thank the financial support from the Young Scholars Development Fund of SWPU (201599010096).

\section{References}

[1] J. Li and J. Yang, "Brief discussion on development of coal seam gas industry in China," SCI-TECH Innocation and Productivity, vol. 8, pp. 20-22, 2011.

[2] J. R. Dempsey, J. K. Patterson, K. H. Coats, and J. P. Brill, "An efficient model for evaluating gas field gathering system design," Journal of Petroleum Technology, vol. 23, no. 9, pp. 1067-1073, 1971.

[3] R. A. Startzman, W. M. Brummett, J. C. Ranney, A. S. Emanuel, and R. M. Toronyi, "Computer combines offshore facilities and reservoir forecasts," Petroleum Engineer International, vol. 49, no. 5, pp. 65-74, 1977.

[4] M. D. Trick, R. Agarwal, J. R. Ammer et al., Gas-Field Deliverability Forecasting: A Coupled Reservoir Simulator and Surface Facilities Model, USDOE Morgantown Energy Technology Center, 1994.

[5] M. L. Litvak and B. L. Darlow, "Surface network and well tubinghead pressure constraints in compositional simulation," in Proceedings of the SPE Reservoir Simulation Symposium, San Antonio, Tex, USA, 1995.

[6] B. K. Coats, G. C. Fleming, J. W. Watts, M. Ramé, and G. S. Shiralkar, "A generalized wellbore and surface facility model, fully coupled to a reservoir simulator," SPE Reservoir Evaluation \& Engineering, vol. 7, no. 2, pp. 132-142, 2004. 
[7] S. Al-Mutairi, E. Hayder, A. Munoz, A. Al-Shammari, and N. Al-Jama, "A study of coupling surface network to reservoir simulation model in a large middle east field," in Proceedings of the North Africa Technical Conference and Exhibition 2010 (NATC '10), pp. 805-814, Cairo, Egypt, February 2010.

[8] B. Guyaguler, V. J. Zapata, H. Cao, H. F. Stamati, and J. A. Holmes, "Near-well-subdomain simulations for accurate inflow-performance- relationship calculation to improve stability of reservoir/network coupling," SPE Reservoir Evaluation \& Engineering, vol. 14, no. 5, pp. 634-643, 2011.

[9] M. H. Cullender and R. V. Smith, "Practical solution of gasflow equations for wells and pipelines with large temperature gradients," Society of Petroleum Engineers, pp. 281-287, 1956.

[10] C. Yang, Gas Extraction Engineering, Petroleum Industry Press, Beijing, China, 2001.

[11] G. Takacs and C. G. Guffey, "Prediction of flowing bottomhole pressures in gas wells," in Proceedings of the SPE Gas Technology Symposium, Dallas, Tex, USA, June 1989.

[12] J. Chen and X. Yue, "Pressure gradient of gas liquid mixture in pumping well," Journal of Daqing. Petroleum Institute, vol. 4, pp. 31-39, 1985.

[13] R. D. Oden and J. W. Jennings, "Modification of the cullender and smith equation for more accurate bottomhole pressure calculations in gas wells," Society of Petroleum Engineers, vol. 10, no. 11, 1988.

[14] A. R. Hasan and C. S. Kabir, "Study of multiphase flow behavior in vertical wells," SPE Production Engineering, vol. 3, no. 2, pp. 263-272, 1988.

[15] X. Liu, Y. Qi, C. Liu, Y. Li, and P. Zhao, "Prediction of flowing bottomhole pressures for two-phase coalbed methane wells," Acta Petrolei Sinica, vol. 31, no. 6, pp. 998-1003, 2010.

[16] H. D. Beggs and J. R. Brill, "Study of two-phase flow in inclined pipes," Journal of Petroleum Technology, vol. 25, pp. 607-617, 1973.

[17] L. Wei, Y. Liu, and Z. Ren, "Node parameter calculation method of oil and gas gathering pipeline network," Journal of Daqing Petroleum Institute, vol. 27, no. 4, pp. 79-83, 2003.

[18] T. Gentzis and D. Bolen, "The use of numerical simulation in predicting coalbed methane producibility from the gates coals, alberta inner foothills, canada: comparison with mannville coal CBM production in the Alberta Syncline," International Journal of Coal Geology, vol. 74, no. 3-4, pp. 215-236, 2008.

[19] C. Yang and Y. Sang, "Application of material balance method in coalbed gas well productivity forecast," Drilling and Production Technology, vol. 23, no. 4, pp. 33-37, 2000.

[20] G. R. King, "Material balance techniques for coal seam and Devonian shale gas reservoirs," in Proceedings of the SPE Annual Technical Conference and Exhibition, pp. 181-192, New Orleans, La, USA, September 1990.

[21] I. Palmer and J. Mansoori, "How permeability depends on stress and pore pressure in coalbeds: a new model," in Proceedings of the SPE Annual Technical Conference and Exhibition, pp. 557565, December 1998.

[22] A. Corey and C. Rathjens, "Effect of stratification on relative permeability," Journal of Petroleum Technology, vol. 8, no. 12, pp. 69-71, 2013.

[23] J. Yang, Y. Wang, and Z. Chen, "Computation of bottom hole flowing pressure (WBHP) and analysis of influencing factors in coalbed methane well," in Proceedings of the Coalbed Methane Conference, pp. 370-377, Beijing, China, 2008. 


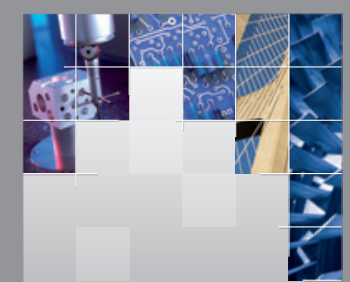

\section{Enfincering}
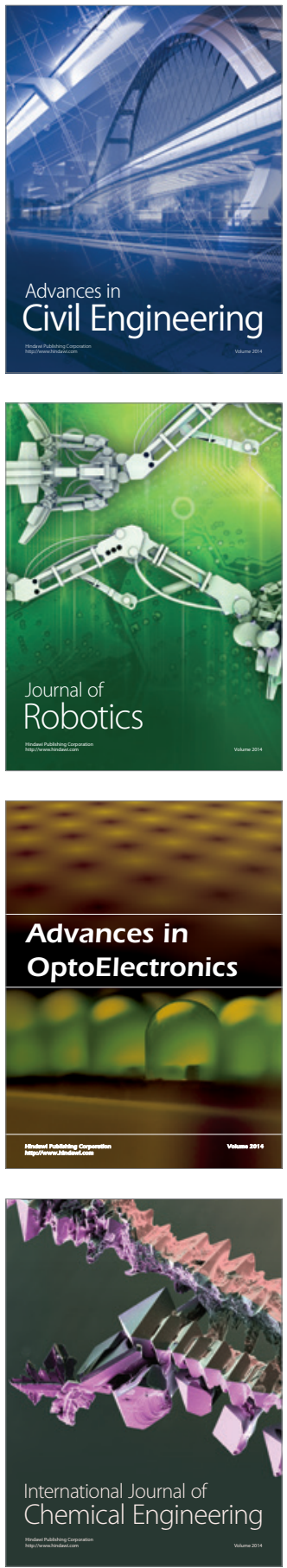

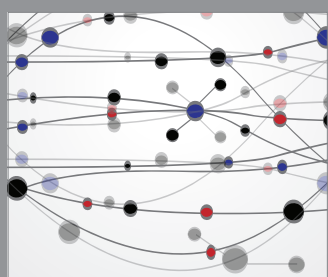

The Scientific World Journal

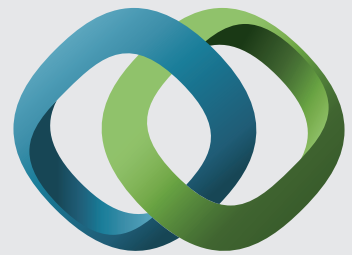

\section{Hindawi}

Submit your manuscripts at

https://www.hindawi.com
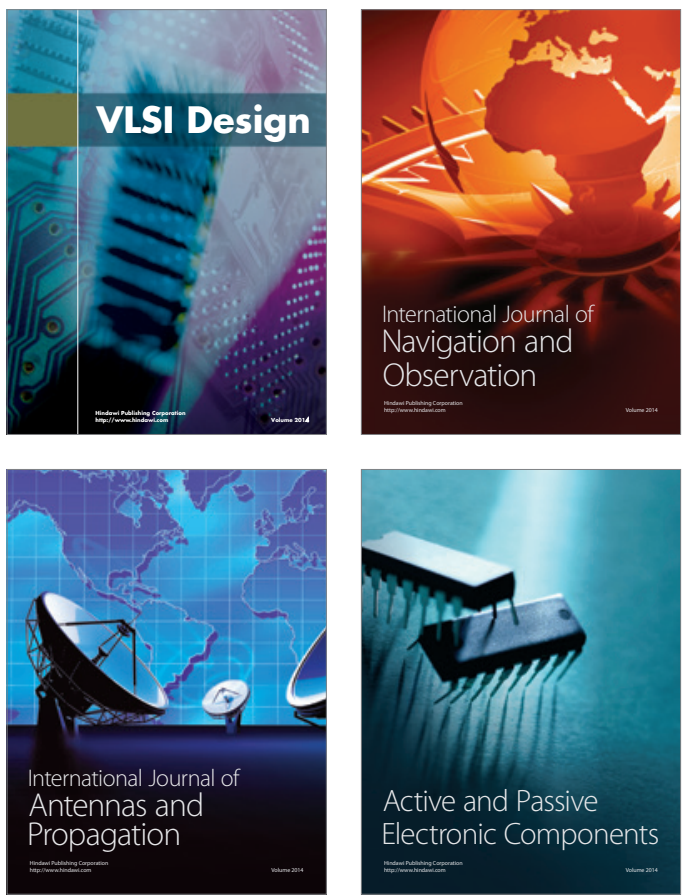
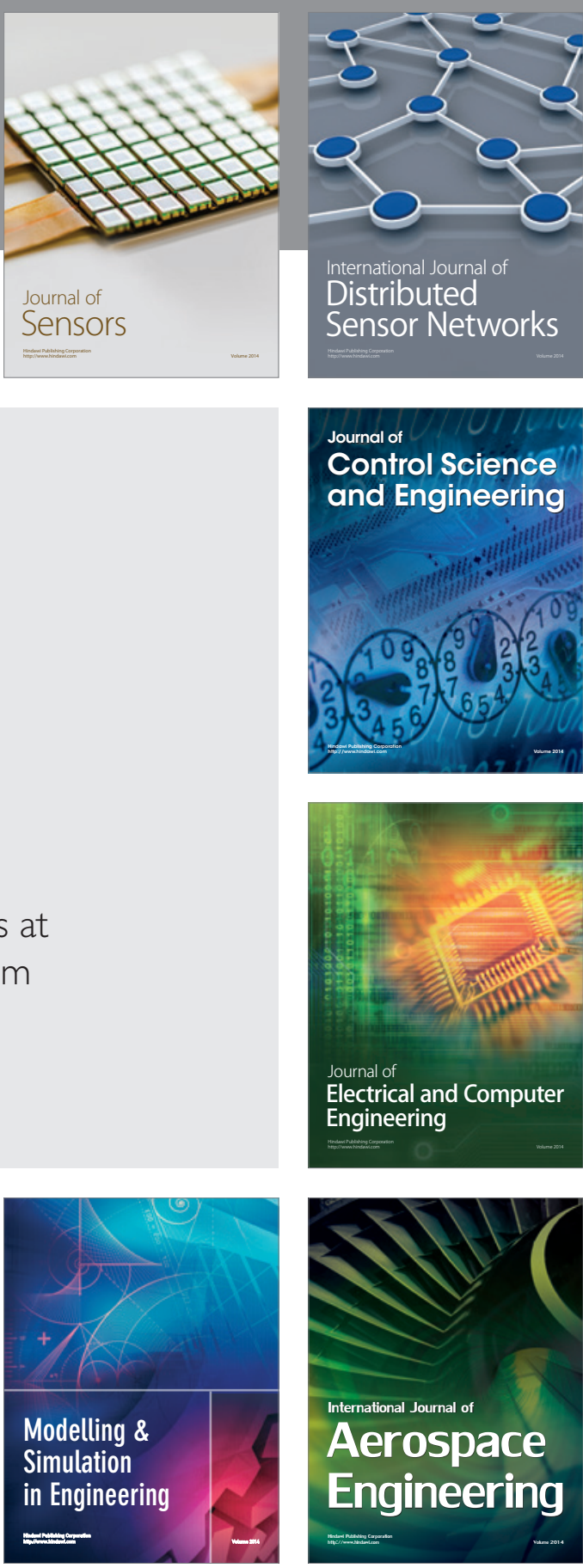

International Journal of

Distributed

Sensor Networks

$-$

Joumal of

Control Science

and Engineering
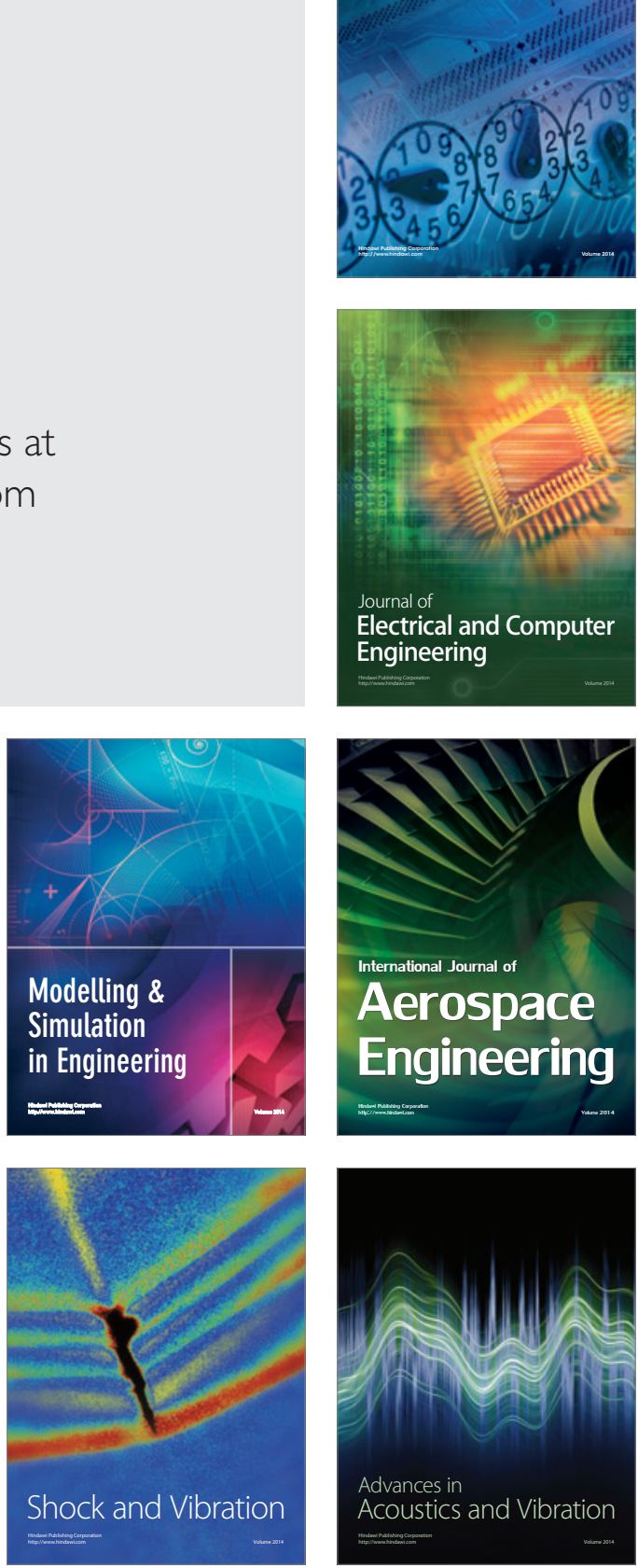